\section{Response of Cybrids and a Somatic Hybrid of Lemon to Phoma tracheiphila Infections}

\author{
Nicasio Tusa ${ }^{1}$ and Sergio Fatta Del Bosco \\ Centro di Studio per il Miglioramento Genetico degli Agrumi, C.N.R., Viale \\ delle Scienze, 90128 Palermo, Italy
}

\section{Franco Nigro and Antonio Ippolito \\ Dipartimento Protezione delle Piante dalle Malattie. Universita' degli Studi di Bari, Via Amendola, 165/a 70126, Bari, Italy}

\section{Additional index words. symmetrical and asymmetrical protoplast fusion, mal secco, Citrus limon, Citrus improvement}

\begin{abstract}
The reaction of lemon hybrids obtained by symmetrical and asymmetrical protoplast fusion, toward "mal secco" infection caused by Phoma tracheiphila (Petri) Kanc. et Ghik. was examined. Resistance was tested in 'Valencia' sweet orange [Citrus sinensis (L.) Osbeck] and 'Femminello' lemon [C. limon (L.) Burm. f.] somatic hybrid and cybrids, by stem and leaf inoculation tests and by analysis of propagule number of $P$. tracheiphila in the xylem of stem-inoculated plants. In general, the somatic hybrid and the cybrids showed an intermediate degree of resistance, with slight differences in disease symptoms, in comparison with resistant 'Monachello' lemon and susceptible 'Femminello' lemon, used as controls. The lower mortality in the asymmetrical lemon cybrids suggests that specific mechanisms of resistance to the disease could be activated in these genotypes.
\end{abstract}

The development of improved Citrus cultivars is an important breeding objective, since many factors limit the market potential for Italian citriculture (e.g., seediness, small fruit size, unfruitfulness, early maturity). For lemon, a high priority is the development of diseaseresistant plants, since most commercial lemon cultivars are seriously damaged by a systemic fungal disease, called "mal secco" and caused by Phoma tracheiphila. The fungus mainly penetrates through wounds and easily colonizes the xylem, inducing withering and dieback of twigs and branches. A sudden wilt of whole branches or trees may also occur when infections take place on the main roots or trunk. The few resistant lemon cultivars ('Monachello' and 'Interdonato') lack the productivity and fruit quality of the susceptible 'Femminello' (Salerno and Cutuli, 1977).

Somatic hybridization via symmetrical and asymmetrical protoplast fusion could represent a primary strategy in obtaining improved disease-tolerant scions and rootstocks (Gmitter et al., 1992; Grosser et al., 1990a, 1990b, 1990c). By symmetrically combining nuclear

Received for publication 9 Mar. 1998. Accepted for publication 18 May 1999. We thankDr.L.W. Timmer (Univ. of Florida, IFAS, Lake Alfred) for useful comments and for a critical reviewing of the manuscript. The cost of publishing this paper was defrayed in part by the payment of page charges. Under postal regulations, this paper therefore must be hereby marked advertisement solely to indicate this fact.

${ }^{1}$ To whom reprint requests should be addressed: Dr. N. Tusa, Centro di Studio per il Miglioramento Genetico degli Agrumi. C.N.R., Viale delle Scienze c/oFacolta' di Agraria, 90128 Palermo, Italy (phone: 3991 423398-484482-6521100; fax: 3991 6521098; e-mail: ntusa@unipa.it). genomes, one can regenerate allotetraploid somatic hybrid plants, which can be used as pollen parents in backcrosses, in efforts to produce triploid cultivars with desirable horticultural traits and disease tolerance. However, in several somatic hybridization experiments, spontaneous elimination of the nucleus of one fusion partner sometimes occurs; as a result, "cybrids," individuals that contain the nucleus of only one partner in combination with some organellar components of the other partner, may be recovered. Direct utilization of such asymmetrical somatic hybrids (cybrids) in commercial orchards may be possible if adequate horticultural characteristics are maintained and disease tolerance is achieved (Grosser et al., 1996; Vardi et al., 1987). The practical value of citrus cybrids is, however, currently unknown since the nuclear/cytoplasmic interactions are still obscure in Citrus.

The objective of this research was to evaluate tolerance toward mal secco infections in the interspecific allotetraploid somatic hybrid plant 'Valencia' sweet orange + 'Femminello' lemon (Tusa et al., 1990) and in two 'Femminello' lemon cybrids (Grosser et al., 1996) that were regenerated following fusion of embryogenic with mesophyll protoplasts of the parents. Evaluation of the mal secco tolerance of the allotetraploid hybrid 'Valencia + Femminello' is extremely important, since it is fertile and could be used in interploid sexual hybridization with diploid 'Femminello' lemon to produce improved seedless triploid lemontypes tolerant to mal secco. Understanding the status of 'Femminello' lemon cybrids would provide new information on the nuclear/cytoplasmic interactions involved in important horticultural traits.
The allotetraploid somatic hybrid 'Valencia + Femminello' and the two 'Femminello' lemon cybrid plants used for this study, one diploid $(2 \mathrm{n}=2 \mathrm{x}=18)$ and one tetraploid $(2 \mathrm{n}=$ $4 \mathrm{x}=36$ ), were produced by polyethylene glycol (PEG)-induced somatic fusion of nucellus-derived embryogenic protoplasts with protoplasts derived from nucellar seedlings, as previously described (Grosser et al., 1996; Tusa et al., 1990). Buds were grafted onto 2year-old sour orange (C. aurantium L.) seedlings in 1989.

Mal secco resistance was assayed by specific tests performed in a growth chamber maintained at $20 \pm 1{ }^{\circ} \mathrm{C}$ and artificially illuminated for $12 \mathrm{~h}$ (Gro-lux tubes, type F4T12/ Gro; Sylvania, Danvers, Mass.) with a light intensity of $100 \mu \mathrm{E}$. The somatic hybrid and cybrid plants were grown in a mixture of soil (70\%) and peat (30\%) in 3-L plastic containers. 'Femminello' and 'Monachello' lemons, highly susceptible and resistant to the disease, respectively, were used as controls. The budwood of 'Monachello' and 'Femminello' lemon was collected from 7-year-old plants.

Stem and leaf inoculation were performed as described by Luisi et al. (1978), since these techniques were effective for evaluating host resistance against the disease, and the results are closely related to the susceptibility to mal secco in mature plants in the groves (De Cicco et al., 1988; Ippolito et al., 1990). Stem inoculation was performed on sour orange rootstock, 4 to $5 \mathrm{~cm}$ above the crown. The inoculum (a drop of conidia adjusted to $10^{6}$ conidia/ $\mathrm{mL}$ ) was introduced into the transpiration stream of 10 replicate plants per genotype by notching the xylem vessels with the blade of a knife on opposite sides of the stem. Leaf inoculation was performed on 6-month-old leaves by pricking the petiole with three entomological pins mounted on a cork and placing a $10-\mu \mathrm{L}$ drop of conidial suspension $\left(10^{7}\right.$ conidia/mL) at each inoculation site (two per leaf). Each treatment was comprised of five plants of each genotype and five leaves were inoculated on each plant.

When the symptoms appeared (20 and $15 \mathrm{~d}$ after inoculation for stem and leaf test, respectively), the development of the disease was assessed approximately weekly, using empirical scales. Severity of symptoms following stem inoculations was rated on a scale of 0 to 5: $0=$ no disease symptoms; $1=$ one or two apical leaves showing vein chlorosis $; 2=<50 \%$ leaves with vein or extended chlorosis, wilted or fallen; $3=>50 \%$ leaves with symptoms or fallen; $4=$ all or almost all leaves wilted or fallen, necrosis of the plant starting from the top. The test was concluded when all plants of the same genotype had reached a score of 4 . Moreover, at the end of the test the number of dead plants was also recorded. Disease severity following leaf inoculations was also rated on a scale of 0 to 5: $0=$ no sign of infection; 1 $=$ chlorotic halo around the inoculation point; $2=$ chlorosis of the vein close to the inoculation point; $3=$ vein chlorosis extending as far as the leaf edge; $4=$ generalized chlorosis and 
/or browning of the veins close to the inoculation point (Luisi et al., 1978). The test was concluded when $90 \%$ of the leaves of a genotype scored 4 . The values of the empirical scale were utilized to compute the McKinney index (McKinney, 1923), by means of the formula:

$$
\mathrm{Mi}=\left[\sum(\mathrm{d} \cdot \mathrm{f}) /(\mathrm{Tn} \cdot \mathrm{D})\right] \cdot 100
$$

where $d$ is the degree of disease intensity assessed on the leaf and $\mathrm{f}$ its frequency; $\mathrm{Tn}$ is the total number of the leaves examined (healthy and diseased); and D the highest degree of disease intensity occurring on the empirical scale. This index represents the weighted average of the disease severity, expressed as actual percentage with respect to the possible maximum level of disease $(100 \%)$.

The number of propagules of the pathogen in the xylem of stem-inoculated lemon plants was evaluated after the last assessment of disease intensity, using the technique of Buchenauer and Erwin (1972) as modified by Lima et al. (1994) for P. tracheiphila. For each genotype, the stems of three plants showing a disease intensity between 3 and 4 were chosen in order to evaluate the rate of xylem colonization at similar degrees of disease symptom intensity. Each plant was separately processed. After aseptically removing the bark, each stem was cut into smaller pieces, and the pieces were weighed and separately homogenized for $3 \mathrm{~min}$ in $100 \mathrm{~mL}$ of cold, sterile water. The homogenate was serially diluted $(1 / 10,1 / 100$, $1 / 1000$ ) and $250 \mu \mathrm{L}$ were mixed in petri dishes with $12 \mathrm{~mL}$ of potato sucrose agar at $50{ }^{\circ} \mathrm{C}$, amended with streptomycin sulfate (250 $\left.\mathrm{mg} \cdot \mathrm{L}^{-1}\right)$. For each stem and dilution, five petri plates were utilized. The number of $P$. tracheiphila colonies were counted after 4 to 6 $\mathrm{d}$ of incubation at $21 \pm 1^{\circ} \mathrm{C}$.

Stem and leaf inoculation tests were arranged in a randomized complete-block design. For stem inoculations, five replicates, each comprising two plants per genotype, were used; for leaf inoculation, five replicates, each comprising one plant with five inoculated leaves, were used. The data were subjected to analysis of variance and the mean values compared using Duncan's multiple range test (Snedecor and Cochran, 1980). The percentage data (i.e., McKinney index) showed high heterogeneity of variance when checked by Bartlett's test; therefore, before analysis, the data were converted into Bliss angular values $(\arcsin \sqrt{ } \%)$, to reduce heterogeneity. The data were then retested and subjected to chi-square normality (Gomez and Gomez, 1984; Little and Hills, 1972).

\section{Results}

Forty-five days after stem inoculation, the allotetraploid somatic 'Valencia + Femminello' and the diploid and tetraploid 'Femminello' cybrids showed a significantly $(P \leq 0.05)$ lower intensity of mal secco disease than did the susceptible 'Femminello' control (Table1). Three months after inoculation, ratings for the allotetraploid somatic hybrid 'Valencia + Femminello' and the tetraploid 'Femminello' cybrid, while numerically in-

Table1. Parameters of mal secco infections on stem and leaf of somatic hybrid and cybrids of lemon. 'Femminello' and 'Monachello' lemon used as control.

\begin{tabular}{lcccccc}
\hline \hline & & \multicolumn{5}{c}{ Genotype } \\
\cline { 3 - 7 } Observation & $\begin{array}{c}\text { Days after } \\
\text { inoculation }\end{array}$ & Femminello & $\begin{array}{c}\text { Diploid } \\
\text { cybrid }\end{array}$ & $\begin{array}{c}\text { Valencia }+ \\
\text { Femminello }\end{array}$ & $\begin{array}{c}\text { Tetraploid } \\
\text { cybrid }\end{array}$ & Monachello \\
\hline Severity of disease & 45 & $2.07 \mathrm{a}^{z}$ & $1.37 \mathrm{c}$ & $1.34 \mathrm{c}$ & $1.69 \mathrm{~b}$ & $1.77 \mathrm{~b}$ \\
& 90 & $3.42 \mathrm{ab}$ & $3.68 \mathrm{a}$ & $3.21 \mathrm{~b}$ & $3.17 \mathrm{~b}$ & $2.33 \mathrm{c}$ \\
No. propagules of Phoma & & & & & & \\
tracheiphila/g of wood & & $10.4 \mathrm{a}$ & $7.6 \mathrm{ab}$ & $3.3 \mathrm{~b}$ & $3.8 \mathrm{~b}$ & $1.7 \mathrm{~b}$ \\
McKinney index values & 20 & $16.9 \mathrm{a}$ & $12.4 \mathrm{~b}$ & $9.5 \mathrm{c}$ & $15.8 \mathrm{a}$ & $2.3 \mathrm{~d}$ \\
& 65 & $91.4 \mathrm{a}$ & $89.4 \mathrm{a}$ & $80.4 \mathrm{a}$ & $80.5 \mathrm{a}$ & $37.1 \mathrm{~b}$ \\
\hline
\end{tabular}

${ }^{\mathrm{z}}$ Mean separation within rows by Duncan's multiple range test, $P \leq 0.05$.

termediate, were not significantly different from that of the susceptible control; 'Monachello' lemon plants showed a significantly lower disease intensity than did the other genotypes tested.

However, differences in the percentages of dead plants between the cybrids and the resistant genotype 'Monachello' were nonsignificant $\left(\chi^{2}=1.44 ; P=0.21\right)$. Only $10 \%$ of the diploid and tetraploid cybrids were dead compared with $40 \%$ of the susceptible 'Femminello' and $20 \%$ of resistant 'Monachello'. The somatic hybrid 'Valencia + Femminello', at $30 \%$ mortality, was intermediate. On the surviving plants of this somatic hybrid, abundant gummy exudates were observed near the leaf scars.

The number of propagules of $P$.tracheiphila per gram of wood was significantly lower $(P \leq$ $0.05)$ on the stems of the somatic hybrid 'Valencia+Femminello' and tetraploid cybrids than on the susceptible 'Femminello' (Table 1). The value for the diploid cybrid, while numerically different, was not statistically different from that for the susceptible control. However, 'Monachello' lemon showed the lowest value.

Twenty days after inoculation, McKinney index values for leaf-inoculated plants of the allotetraploid somatic hybrid 'Valencia + Femminello' and the diploid 'Femminello' lemon cybrid were significantly lower than that for the susceptible control (Table 1). The resistant 'Monachello' genotype had the lowest value. However, at the end of the test ( 65 d after inoculation) differences among the somatic hybrid, both cybrids, and the 'Femminello' susceptible control were nonsignificant.

\section{Discussion}

In the present work, the resistance to $P$. tracheiphila infections was tested in three different genotypes, the amphidiploid somatic hybrid 'Valencia + Femminello' and two 'Femminello' lemon cybrids, obtained by symmetrical and asymmetrical protoplast fusion, respectively. The value of fertile somatic hybrids in Citrus scion improvement is known, since they can be used in interploid crosses with diploid cultivars to obtain triploid seedless plants for selection (Grosser and Gmitter, 1990a, 1990b; Soost and Cameron, 1975). Yet, the practical value of citrus cybrids is currently unknown, because no horticulturally important traits are known to be encoded by organelle DNA (Grosser et al., 1996).

Considering the results obtained in the stem inoculation test, a slower development of the disease in the initial stages seems to be a peculiar trait of the somatic hybrid 'Valencia + Femminello' and both the lemon cybrids, as compared with the susceptible control, while, in the leaf inoculation test, only the somatic hybrid 'Valencia + Femminello' and the diploid lemon cybrid show a slower initial symptom progression. However, compared with the behavior of the resistant 'Monachello' lemon, the somatic hybrid and both cybrids showed a higher intensity of disease symptoms at the end of the various tests, except for the propagule numbers of $P$. tracheiphila in the xylem of stem-inoculated plants. In this case, a low number of propagules was found in the xylem of the somatic hybrid 'Valencia +Femminello', the tetraploid cybrid, and the resistant control. An intermediate reaction was observed in the diploid cybrid with respect to the susceptible and resistant controls. This result may indicate that this character is connected to the observed slower disease development. In 'Monachello' lemon the resistance to mal secco has been characterized and its expression is related to gum reactions, which delay the progress of the pathogen in vascular tissue and allow the plants to compartmentalize the infection (Perrotta et al., 1980; Somma et al., 1979). A similar mechanism could be hypothesized for the somatic hybrid 'Valencia + Femminello', since abundant gummy exudates were observed near the leaf scars of the surviving plants. Our data on propagules of the pathogen in the xylem are in agreement with others (Magnano di San Lio et al., 1992), indicating that extensive vascular colonization is a prerequisite for symptom expression in mal secco-infected sour orange seedlings. In fact, the difference in disease intensity observed on 'Femminello' and 'Monachello' seems to be positively related to the propagule numbers of the pathogen in the xylem. The low percentages of dead plants recorded for diploid and tetraploid lemon cybrids could confirm this hypothesis. Moreover, the lower percentage of dead plants in both 'Femminello' lemon cybrids than in 'Monachello' lemon suggests that mechanisms of resistance to $P$. tracheiphila invasion into the xylem could be activated as an effect of nuclear/cytoplasmic DNA interactions; in these genotypes, the mitochondrial genome is derived from the resistant 'Valencia' sweet orange and the nuclear genome from the susceptible 'Femminello' lemon. 
The allotetraploid somatic hybrid 'Valencia + Femminello' was developed in an effort to combine the good quality and performance of 'Femminello' lemon with the cold hardiness and mal secco tolerance of 'Valencia' (Tusa et al., 1990). In our experiment, disease developed more slowly in 'Valencia + Femminello' in the initial stages; moreover, the lower rate of propagules in the xylem and the lower percentage of dead plants as compared with the susceptible 'Femminello' would indicate an intermediate degree of resistance to mal secco infection. The performance is not surprising, since this genotype is an amphidiploid somatic hybrid obtained from symmetrical protoplast fusion, in which the complete nuclear genomes of both parents were combined. The additive process offers the advantage of transferring important traits for which expression is controlled by complex gene systems; as a result, somatic hybrids may retain many of the attributes of both parents (Grosser et al., 1995). The behavior to $P$. tracheiphila infections seems to be confirmed by preliminary field observations; the somatic hybrid 'Valencia + Femminello' and both of the lemon cybrids have been in experimental field trials for lemon since 1990 to evaluate their horticultural performance and their resistance or tolerance to natural infection of mal secco. To date, all three exhibit adequate horticultural characteristics, being vigorous and having a higher degree of tolerance to mal secco infection than the original 'Femminello' lemon. Complete evaluation of these genotypes is, however, still in progress and is a very long-term and complex effort. Moreover, 'Valencia + Femminello' has confirmed its great potential in citrus cultivar development; in fact, it has been used as a male parent in interploid backcrosses with diploid 'Femminello' lemon, and a large triploid progeny has been rescued (Tusa et al., 1996).

This is the first report in which somatic hybrids and cybrids of lemon have been tested for their resistance to mal secco infections. However, the characterization of the observed resistance (i.e., anatomical and functional char- acters of the xylem, gummy reactions, etc.) deserves further investigation.

\section{Literature Cited}

Buchenauer, H. and D.C. Erwin. 1972. Control of Verticillium wilt of cotton by spraying with acidic solutions of benomyl, methyl 2-benzimidazole carbamate, and thiabendazole. Phytopathol. Z. 75:124-129.

De Cicco, V., A. Ippolito, and M. Salerno. 1988. Response to mal secco infections of two putative resistant lemon lines under controlled environment. p. 99-103. In: Proc. $2^{\text {nd }}$ Intl. Mtg. Mediterranean Tree Crops. Chania, Crete, Greece, 2-4 Nov. 1988

Gmitter, F.G., Jr., J.W. Grosser, and G.A. Moore 1992. Citrus, p. 335-370. In: R.E. Litz and F. Hammerschlag (eds.). Biotechnology of perennial fruit crops. CAB Intl., Oxon, U.K.

Gomez, K.A. and A.A. Gomez. 1984. Statistical procedures for agricultural research. $2^{\text {nd }}$ ed. Wiley, New York.

Grosser, J.W. and F.G. Gmitter, Jr. 1990a. Protoplast fusion and citrus improvement. Plant Breed. Rev. 8:339-374.

Grosser, J.W. and F.G. Gmitter, Jr. 1990b. Wide hybridization of citrus via protoplast fusion: Progress, strategies and limitations, p. 31-41. In: A.B. Bennet and S.D. O'Neil (eds.). Horticultural biotechnology, plant biology. vol. 11 . Wiley-Liss, New York.

Grosser, J.W. and F.G. Gmitter, Jr. 1990c. Somatic hybridization of citrus with wild relatives for germplasm enhancement and cultivar development. HortScience 25:147-151.

Grosser, J.W., F.G. Gmitter, Jr., W.S. Castle, and J.L. Chandler. 1995. Production and evaluation of citrus somatic hybrid rootstocks: Progress report. Proc. Fla. Hort. Soc. 108:140-143.

Grosser, J.W., F.G. Gmitter, Jr., N. Tusa, G Reforgiato Recupero, and P. Cucinotta. 1996. Further evidence of a cybridization requirement for plant regeneration from citrus leaf protoplasts following somatic fusion. Plant Cell Rpt. 15:672-676.

Ippolito, A., V. De Cicco, and E. Traversa. 1990. Behaviour of some grafted lemon rootstocks towards mal secco infections in controlled enviroment. XXIII Intl. Hort. Congr., Florence, Abstr. p. 512.

Lima, G., F. Nigro, A. Santomauro, and A. Ippolito. 1994. Ulteriori tentativi di lotta biologica contro il mal secco degli agrumi mediante isolati ipovirulenti del patogeno. La Difesa delle piante 17:135-144.

Little, T.M. and F.J. Hills. 1972. Statistical methods in agricultural research. Univ. of California, Davis.

Luisi, N., V. De Cicco, G. Cutuli, and M. Salerno. 1978. Factors in early testing for citrus mal secco resistance. Proc. Intl. Soc. Citricult. 1:197200

Magnano di San Lio, G., S.O. Cacciola, A. Pane, and S. Grasso. 1992. Relationship between xylem colonization and symptom expression in mal secco infected sour orange seedlings. Proc. Intl. Soc. Citricult. 2:873-876.

McKinney, H.H. 1923. Influence of soil temperature and moisture on infection of wheat seedlings by Helminthosporium sativum. J. Agr. Res. 26:195-218.

Perrotta, G., G. Magnano di San Lio, and M. Bassi. 1980. Some anatomical and morpho-functional aspect of resistance to Phoma tracheiphila in citrus plants. Phytopathol. Z. 98:346- 358

Salerno, M. and G. Cutuli. 1977. Control of citrus mal secco in Italy today. Proc. Intl. Soc. Citricult. 3:1001-1003

Snedecor, G.W. and W.G. Cochran, 1980. Statistical methods. The Iowa State Univ. Press, Ames.

Somma, V., G. Cutuli, and M. Salerno. 1979. Ricerche sul saggio della resistenza al mal secco di giovani limoni innestati, p. 71-80. In: Proc. secondo seminario di studio sul miglioramento genetico del limone. Giovinazzo, Bari, Italy, 56 Apr. 1979.

Soost, R.K. and J.W. Cameron. 1975. Citrus, p. 507-540. In: J. Janick and J.N. Moore (eds.). Advanced fruit breeding. Purdue Univ. Press, West Lafayette, Ind.

Tusa, N., S. Fatta Del Bosco, L. Nardi, and S. Lucretti. 1996. Obtaining triploid plants by crossing citrus lemon cv. 'Femminello' $2 \mathrm{n} \mathrm{x} \mathrm{4n} \mathrm{al-}$ lotetraploid somatic hybrids. Proc. Intl. Soc. Citricult. 1:133-136.

Tusa, N., J.W. Grosser, and F.G. Gmitter, Jr. 1990. Plant regeneration of 'Valencia' sweet orange, 'Femminello' lemon, and the interspecific somatic hybrid following protoplast fusion. J. Amer. Soc. Hort. Sci. 115:1043-1046.

Vardi, A., A. Breiman, and E. Galun. 1987. Citrus cybrids: Production by donor-recipient protoplast fusion and verification by mitochondrial restriction profiles. Theor. Appl. Genet. 75:5158. 